\title{
SPECT/CT Assessment of Infected Intracardiac Devices With and Without Attenuation Correction
}

Lee C. Morris ${ }^{1}$ and Marques L. Bradshaw ${ }^{2}$

${ }^{1}$ MUSC College of Medicine, Medical University of South Carolina, Charleston, South Carolina; and ${ }^{2}$ Department of Radiology, Medical University of South Carolina, Charleston, South Carolina

Left ventricular assist devices (LVADs) provide the ability to maintain cardiac output and sustain life as a bridge to transplantation, definitive therapy, or a permanent decision. We present a case of LVAD drive infection that was differentiated from pump infection by the use of attenuation-corrected and non-attenuationcorrected CT, along with correlation with the planar images. Clinically, the patient was suspected of having infection; however, the clinician did not know which components of the device were involved. The patient's scan showed abnormal activity along the driveline with and without attenuation correction, whereas the pump showed abnormal activity with attenuation correction only. This finding suggested that the drive line was infected but that the activity within the pump was secondary to overcorrection of attenuation. The driveline was cultured, confirming infection.

Key Words: infectious disease; quality assurance; SPECT/CT; attenuation correction; LVAD

J Nucl Med Technol 2016; 44:94-95

DOI: 10.2967/jnmt.115.156844

$\mathbf{T}$ he purpose of this teaching case study is to demonstrate the use of both attenuation-corrected and non-attenuationcorrected SPECT images when assessing for infection of intracardiac devices, in particular left ventricular assist devices (LVADs). LVADs provide the ability to maintain cardiac output and sustain life as a bridge to transplantation, definitive therapy, or a permanent decision. Potential complications include bleeding, infection, and thromboembolism. The degree and extent of infection can be difficult to discern by clinical measures, leading to imaging. Infections that involve the pump pocket can be particularly worrisome, as the best way to clear the infection would be to remove the pump. We present a case of LVAD drive infection that was differentiated from pump infection by the use of attenuation-corrected and nonattenuation-corrected CT, along with correlation with the planar images.

Received Mar. 2, 2015; revision accepted Jun. 25, 2015.

For correspondence or reprints contact: Marques L. Bradshaw, Medical University of South Carolina, Department of Radiology, 96 Jonathan Lucas St., MSC 323, Charleston, SC 29425.

E-mail: bradsham@musc.edu

Published online Aug. 13, 2015.

COPYRIGHT (C) 2016 by the Society of Nuclear Medicine and Molecular Imaging, Inc.

\section{CASE REPORT}

A 61-y-old man with ischemic cardiomyopathy and New York Heart Association class II congestive heart failure after LVAD placement presented with drainage from his drive line exit site and left upper quadrant pain creating concern about infection. The exudate was cultured, and a ${ }^{67} \mathrm{Ga}$ scan was ordered to determine the etiology of his left upper quadrant pain and the extent of infection.

After administration of $1.9 \mathrm{MBq}(5.2 \mathrm{mCi})$ of ${ }^{67} \mathrm{Ga}$, whole-body and multiple planar views were obtained at $48 \mathrm{~h}$. SPECT/CT images of the lower chest and upper abdomen were obtained for better anatomic localization. The planar images demonstrated abnormal curvilinear activity (Fig. 1) along the suspected course of the LVAD drive line site, which was confirmed by SPECT/CT findings showing abnormal activity along the drive line with and without attenuation correction. The SPECT/CT scan also demonstrated intense activity within the LVAD pump. This activity was not expected as it was not seen on the planar images. The nuclear medicine physician reviewed the non-attenuation-corrected images, which showed no abnormal activity within the LVAD pump (Fig. 2). The observations from the SPECT/CT study were interpreted as consistent with infection of the LVAD driveline only. The cultured exudate was pos-

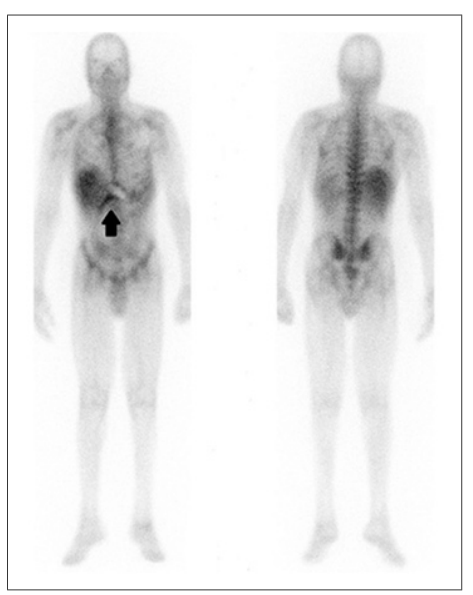

FIGURE 1. Planar anterior and posterior images from whole-body ${ }^{67} \mathrm{Ga}$ scan demonstrate curvilinear activity inferior to rib cage near liver (arrow). itive for Acinetobacter baumannii. Infection of the LVAD pump itself was not definitively excluded since the LVAD pump was not cultured but was unlikely given the absence of activity on nonattenuation-corrected and planar images.

\section{DISCUSSION}

Implanted intracardiac devices present a unique challenge in terms of diagnosing the presence of infection. The clinical presentation varies 


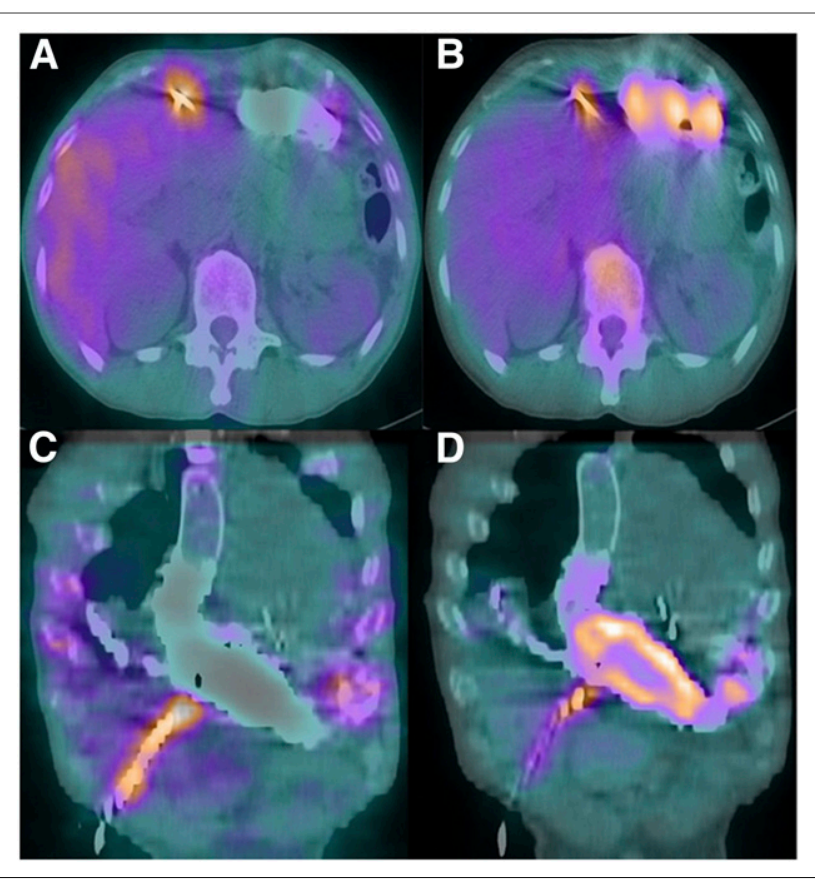

FIGURE 2. Axial SPECT without (A) and with (B) attenuation correction images, and corresponding coronal SPECT without (C) and with (D) attenuation correction. Increased activity is seen on all images within drive line, whereas pump demonstrates activity on attenuation correction images only.

depending on the type of device implanted, the causative organism, the location (generator or pump pocket, subcutaneous leads, endovascular leads), and whether there is concomitant bacteremia (1). In particular, LVADs have a higher incidence of infection secondary to their large surface area, varied construction material, and crossing of several tissue planes and organ spaces $(1) .{ }^{67} \mathrm{Ga}$ and white blood cell studies offer the ability to diagnose the presence and extent of infection in a noninvasive manner (2). SPECT/CT has made it possible to determine the precise location of infection by eliminating overlap from adjacent structures and showing excellent anatomic detail. Although CT attenuation correction has the benefit of improved localization, there remains the possibility of overcorrection when imaging traverses very dense structures, resulting in an incorrectly perceived increase in radiotracer uptake (3). Therefore, this phenomenon could lead to overestimation of the extent of disease. Inaccurate details about the site of infection may lead to improper therapy, as the treatment algorithms depend on the site of infection. Attenuation correction errors with PET/CT have been previously described when dense structures are concerned; however, this phenomenon has not been described clinically with SPECT/CT (4-O).

\section{CONCLUSION}

SPECT/CT has improved the ability to localize findings on planar images; however, this teaching case study demonstrates the importance of correlating the planar images with both non-attenuation-corrected and attenuation-corrected SPECT/CT to increase the accuracy with which infections of intracardiac devices are diagnosed. Further investigations of the effectiveness of having both attenuation-corrected and non-attenuation-corrected images available at the time of image review are ongoing.

\section{DISCLOSURE}

No potential conflict of interest relevant to this article was reported.

\section{REFERENCES}

1. Yesenosky GA, Sinner SW, Kutalek SP, Tunkel AR. Infections of intracardiac devices. In: Manivannan G, ed. Disinfection and Decontamination Principles, Applications and Related Issues. Boca Raton, FL: CRC Press; 2008:373-375.

2. Litzler PY, Manrique A, Etienne M, et al. Leukocyte SPECT/CT for detecting infection of left-ventricular-assist devices: preliminary results. J Nucl Med. 2010;51;1044-1048.

3. Patton JA, Turkington TG. SPECT/CT physical principles and attenuation correction. J Nucl Med Technol. 2008;36:1-10.

4. Abella M, Alessio AM, Mankoff DA, et al. Accuracy of CT-based attenuation correction in PET/CT bone imaging. Phys Med Biol. 2012;57:2477-2490.

5. Dizendorf E, Hany TF, Buck A, von Schulthess GK, Burger C. Cause and magnitude of the error induced by oral CT contrast agent in CT-based attenuation correction of PET emission studies. J Nucl Med. 2003;44:732-738.

6. Kamel EM, Burger C, von Schulthess GK, Goerres GW. Impact of metallic dental implants on CT-based attenuation correction in a combined PET/CT scanner. Eur Radiol. 2003;13:724-728. 